the complications, hospital stay and number of radiological procedures done is significantly reduced when compared to percutaneous drainage alone. These results are encouraging and very important for those centre's that have been treating WOPN by the percutaneous route alone. Addition of transmural drainage to standard percutaneous drainage in this study eliminated the risk of development of external pancreatic fistulae as well as surprisingly reduced the incidence of pseudoaneurysmal bleed, possibly because of better pancreatic drainage in the combined group. Although an interesting study, the retrospective nature is a limitation of this study and these results need to be confirmed in multicenter, prospective randomized trials. We also need to look at the long term follow up of these patients, especially the ones with the disconnected pancreatic duct. Moreover, studies are also needed that compare this combined treatment protocol with surgical or endoscopic necrosectomy alone. It will be very interesting to find out what additional advantage the percutaneous drainage will have over and above endoscopic drainage alone.

\section{Large Balloon Dilation vs Mechanical Lithotripsy for the Management of Large Bile Duct Stones: A Prospective Randomized Study}

\section{G. Stefanidis, N. Viazis, D. Pleskow, S. Manolakopoulos, L. Theocharis, C. Christodoulou, N. Kotsikoros, J. Giannousis, S. Sgouros, M. Rodias, A. Katsikani, R. Chuttani}

Am J Gastroenterol early online 2 Nov 2010 doi: 10.1038/ ajg. 2010.421

Endoscopic common bile duct (CBD) stone removal is difficult in patients with large stones $(>12 \mathrm{~mm})$, multiple stones, barrel-shaped stones, and tapering course of CBD. Traditionally these patients have been either treated surgically or endoscopic removal of these stones has been attempted after mechanical lithotripsy (ML). Advent of endoscopic large balloon dilatation (LBD) of the papilla following endoscopic sphincterotomy (EST) has offered an alternative for removal of difficult CBD stones. However, the initial enthusiasm with this technique of LBD dampened because of reports of increased risk of pancreatitis. [3] Despite this risk of pancreatitis, LBD is an effective alternative for management of patients with difficult CBD stones. The authors of the current study therefore conducted a prospective randomized controlled trial to compare the therapeutic benefits and complications of EST followed by LBD or ML for the management of large bile duct stones.

A total of 90 patients with large bile duct stones (12 - 20 $\mathrm{mm})$ were randomized to EST followed by $\operatorname{LBD}(n=45)$ or EST followed by ML $(n=45)$ [no significant difference in the stone size as well as the CBD diameter between the two groups]. Randomization was performed using the sealed envelope technique and all the ERCP's were performed by a single operator. Patients needing precut for CBD access, with stones more than $20 \mathrm{~mm}$ or with accidental pancreatogram were excluded from the study. In both the groups, complete EST was accomplished by extending the cut up to the major horizontal fold crossing the intramural portion of the bile duct. The patients in the LBD group underwent balloon dilatation with CRE Esophageal / Pyloric balloon, maximum diameter 15, 18, or $20 \mathrm{~mm}$; length $5 \mathrm{~cm}$. (Boston Scientific, Natick, MA). The size of the balloon used was chosen according to the diameter of the bile duct stones and care was taken that it should not exceed the maximum diameter of the bile duct. The balloons were gradually inflated till the waist was obliterated and after that the balloons remain inflated for 10 to 12 seconds. The mechanical lithotripsy was done using a mechanical lithotriptor (BML 4Q, Lithocrush 201 or 202Q; Olympus Optical).

Out of 45 patients in the LBD group, complete CBD clearance was possible in 44 patients $(97.7 \%)$ whereas complete CBD clearance was possible in 41 out of 45 (91.1\%) patients in ML group and this difference was not statistically significant. A subgroup analysis, taking into account the bile duct stone size also did not reveal a statistical significant difference in the success rate between the two groups. Presence of a very tortuous CBD resulted in failure of removal of CBD stones in the LBD group whereas failure in ML group was due to basket being broken on a hard stone (1 patient) or inability to capture the stone (3 patients). Significantly more number of complications were noted in the ML group in comparison to the LBD group (9 vs. 2 patients; $p=0.049$ ). One patient in each group developed post-ERCP pancreatitis which was mild and self-limiting and was managed conservatively. Six patients in the ML group developed cholangitis whereas none in the LBD group developed cholangitis $(p=0.026)$. Post ERCP bleeding was noted in one patient in each group. One patient in ML group developed CBD perforation whereas none of the patients in LBD group developed it.

\section{Commentary}

The authors of the current study have shown that EST followed by LBD is equally effective as EST followed by ML for the removal of large bile duct stones, but is associated with fewer complications. This is a prospective randomized study that has shown that LBD is safe and effective and the risk of pancreatitis is low (a feared side effect that has limited its use). The authors believe that the low risk of pancreatitis in their study could be because of the expertise of the endoscopists performing the ERCPs, as well as by the fact that the authors excluded patients at increased risk of pancreatitis ( $>5$ accidental pancreatograms or use of,needle knife precutting). 
Also some other studies have also shown that LBD is safe with low risk of pancreatitis as the biliary and pancreatic orifices are separated by previously performed EST.[4] The authors also left the balloon inflated for 10 to 12 seconds only after obliteration of the waist and they believed that this shorter time of inflation could also have decreased the risk of pancreatitis. However, this hypothesis is contrary to an earlier published randomized study comparing 1 minute vs. 5 minute of endoscopic balloon dilatation of intact biliary sphincter where the authors have shown that 5 minute balloon dilatation is associated with better efficacy of stone extraction and reduced risk of pancreatitis. [5] The authors of this study hypothesized that post-ERCP pancreatitis is primarily related to postprocedure papillary edema and outflow obstruction rather than intraprocedural occlusion of the pancreatic sphincter by the balloon and therefore dilations of up to 1 minute, as is typically done, are inadequate and produce excessive postprocedure edema.

Also, the authors have demonstrated that ML is associated with more risk of complications like cholangitis when compared to LBD where none of the patients developed cholangitis. The authors believed that higher cholangitis rate in patients in ML group could be because of trauma to the CBD wall by the lithotriptor wires as well as edema at the sphincterotomy site and / or inadequate sphincterotomy could also contribute to the higher cholangitis rate. The current prospective randomized study has shown that LBD is safe and effective and is associated with fewer complications than $\mathrm{ML}$ and importantly the risk of pancreatitis is low and comparable between the two groups.

\section{References}

1. Ross A, Gluck M, Irani S, et al. Combined endoscopic and percutaneous drainage of organized pancreatic necrosis. Gastrointest Endosc 2010;71:79-84.

2. Rana SS, Bhasin DK. Endoscopic therapy for organized pancreatic necrosis: are we as endoscopists organized? Trop Gastroenterol 2005;26:173-7.

3. Disario JA, Freeman ML, Bjorkman DJ, et al. Endoscopic balloon dilation compared with sphincterotomy for extraction of bile duct stones. Gastroenterology 2004;127:1291-9.

4. Attam R, Freeman ML. Endoscopic papillary balloon dilation for stone extraction: if, when, and for how long? Gastrointest Endosc 2010; 72: 1163-6.

5. Liao WC, Lee CT, Chang CY, Leung JW, Chen JH, Tsai MC, Lin JT, Wu MS, Wang HP. Randomized trial of 1-minute versus 5-minute endoscopic balloon dilation for extraction of bile duct stones. Gastrointest Endosc 2010;72:1154-62.

Correspondence and reprint requests:

Dr. Surinder Singh Rana

Department of Gastroenterology, Post Graduate Institute of Medical

Education and Research (PGIMER), Sector 12, Chandigarh, India Tel:

+91-172-2749123; Fax: +91-172-2744401

E-mail: drsurinderrana@yahoo.co.in

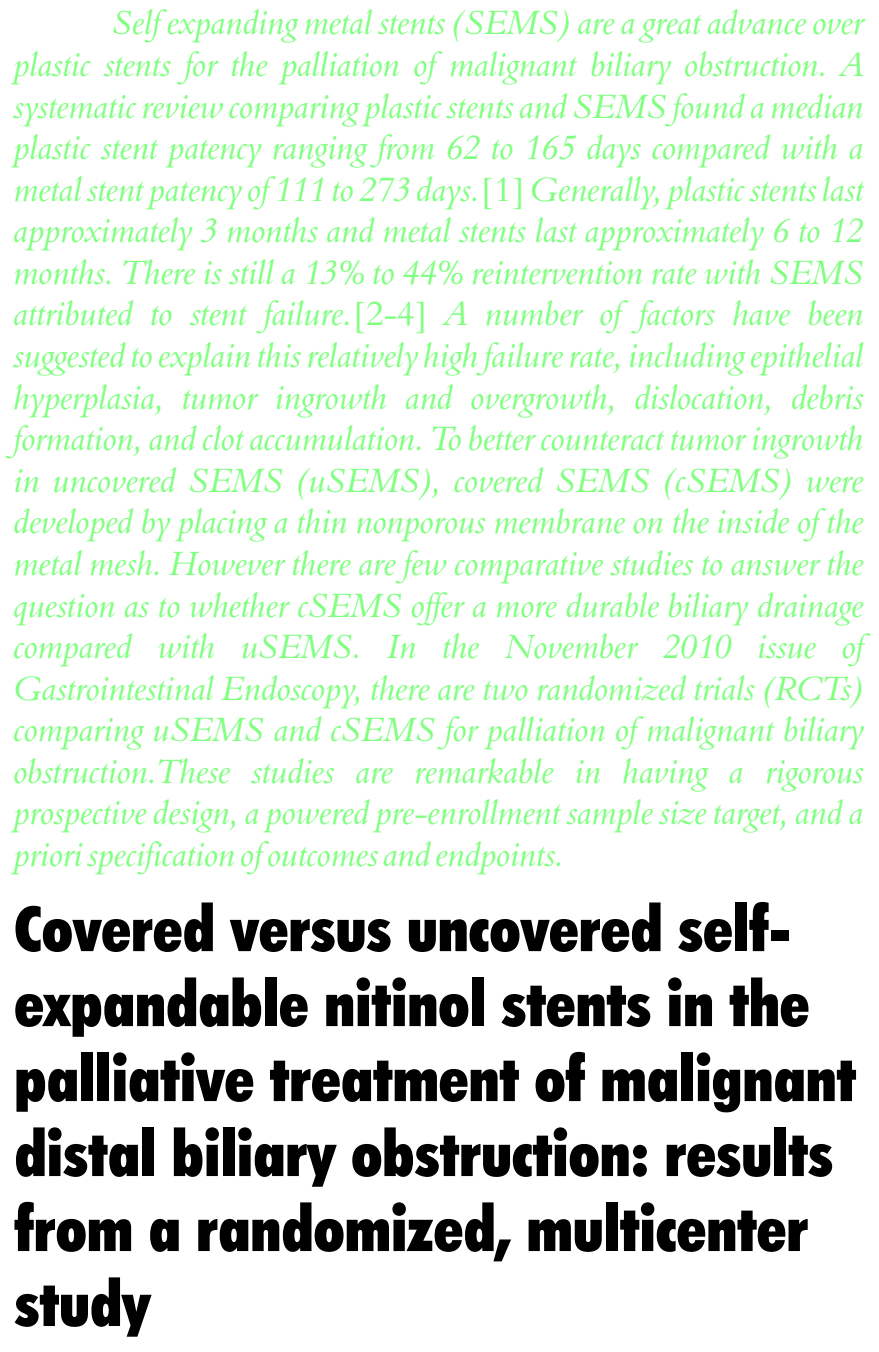

Kullman E, Frozanpor F, Söderlund C, Linder S, Sandström $P$, Lindhoff-Larsson A, Toth $E$, Lindell $G$, Jonas $E$, Freedman J, Ljungman $M$, Rudberg C, Ohlin B, Zacharias R, Leijonmarck CE, Teder K, Ringman A, Persson G, Gözen M, Eriksson O.

Gastrointest Endosc 2010;72:915-23.

The first study was a RCT conducted to compare differences in stent patency, patient survival, and complication rates between covered and uncovered nitinol stents. in patients with malignant biliary obstruction. This multicenter trial was conducted between January 2006 and October 2008, at 10 sites in Sweden. A total of 21 endoscopists with 4 to 25 years of experience performing ERCP participated.

The randomization process, was done in blocks of 20 (10:10) when the patient was in the ERCP suite and after the guidewire had passed the stenosis. The endoscopist opened an opaque sealed envelope with computer-generated random numbers. A total of 400 patients with unresectable distal 